\title{
FOURIER/LAPLACE TRANSFORMS AND RUIN PROBABILITIES
}

\author{
BY \\ FÁtima D.P. LIMA ${ }^{1}$, JORGE M.A. GARCIA ${ }^{2}$ AND \\ AlFrEDo D. EGÍdIO DOS REIS ${ }^{3}$
}

\begin{abstract}
In this paper we use Fourier/Laplace transforms to evaluate numerically relevant probabilities in ruin theory as an application to insurance. The transform of a function is split in two: the real and the imaginary parts. We use an inversion formula based on the real part only, to get the original function.

By using an appropriate algorithm to compute integrals and making use of the properties of these transforms we are able to compute numerically important quantities either in classical or non-classical ruin theory. As far as the classical model is concerned the problems considered have been widely studied. In what concerns the non-classical model, in particular models based on more general renewal risk processes, there is still a long way to go. In either case the approach presented is an easy method giving good approximations for reasonable values of the initial surplus.

To show this we compute numerically ruin probabilities in the classical model and in a renewal risk process in which claim inter-arrival times have an Erlang(2) distribution and compare to exact figures where available. We also consider the computation of the probability and severity of ruin in the classical model.
\end{abstract}

\section{KEYWORDS}

Risk theory, Ruin theory, Fourier transform, Laplace transform, Probability of ultimate ruin, Severity of ruin, Erlang(2) risk process.

1 SGF, Sociedade Gestora de Fundos de Pensões, Lisbon. flima@sgf.pt.

2 CEMAPRE, ISEG, Department of Mathematics, Technical University of Lisbon and SGF, Sociedade Gestora de Fundos de Pensões, Lisbon. jorgegarcia@sgf.pt. Support from Fundação para a Ciência e a Tecnologia - FCT/POCTI is gratefully acknowledged.

3 CEMAPRE and ISEG, Department of Mathematics, Technical University of Lisbon; Rua do Quelhas 6, 1200-781 Lisboa, Portugal. Tel.: +351-213925867. Fax: +351-213922781. alfredo@iseg.utl.pt. Support from Fundação para a Ciência e a Tecnologia - FCT/POCTI is gratefully acknowledged. 


\section{INTRODUCTION}

In this work we consider a continuous time risk process, where claims occur as a renewal process. Times between claims (and the time until the first claim) form a sequence of independent and identically distributed random variables, denoted $\left\{T_{j}\right\}_{j=1}^{\infty}$, with density function $k(\bullet)$ and distribution function $K(\bullet)$, with $K(0)=0$. We denote the claim number occurrence process as $\{N(t), t \geq 0\}$, which we will consider as a renewal process. $N(t)$ represents the number of claims in the interval $(0, t]$.

Let $\left\{X_{j}\right\}_{j=1}^{\infty}$ be a sequence of independent and identically distributed random variables, where $X_{j}$ denotes the amount of the $j$-th claim. $\left\{X_{j}\right\}$ and $\left\{T_{k}\right\}$ are independent. Let $P\left({ }^{\circ}\right)$ and $p(\bullet)$ be the distribution and density function of $X_{j}$, respectively, with $P(0)=0$. We assume that the means of $X_{j}$ and $T_{k}$ exist and denote $p_{1}=E\left[X_{j}\right]$. Let $c$ denote the insurer's premium income per unit time. We will assume that

$$
c E\left[T_{j}\right]>E\left[X_{j}\right] \forall j
$$

Let $\{U(t), t \geq 0\}$ be the surplus or risk process such that

$$
U(t)=u+c t-\sum_{j=1}^{N(t)} X_{j}
$$

where $u$ is the initial surplus and define the time until ruin, denoted $T$, by

$$
T=\left\{\begin{array}{l}
\inf \{t: U(t)<0\} \\
\infty \text { if } U(t) \geq 0 \forall t
\end{array} .\right.
$$

The surplus at the time of $n$-th claim is,

$$
U\left(\sum_{j=1}^{n} T_{j}\right)=u+\sum_{j=1}^{n}\left(c T_{j}-X_{j}\right)
$$

The probability of ultimate ruin from initial surplus $u$ for this risk process is defined as

$$
\psi(u)=\operatorname{Pr}\left\{u+\sum_{j=1}^{n}\left(c T_{j}-X_{j}\right)<0 \text { for some } n, n=1,2, \ldots\right\}=\operatorname{Pr}\{T<\infty \mid U(0)=u\},
$$

and let $\delta(u)=1-\psi(u)$ denote the survival probability. Note that if the event ruin is to happen this will occur at the time of a claim occurrence. If the moment generating function of $X_{j}$ exists, in an appropriate open interval, then the adjustment coefficient for this risk process is the unique positive 
number $R$ such that [see for instance Sparre Andersen (1957) or Dickson and Hipp (1998) as a recent reference]

$$
E\left[e^{-c R T_{j}}\right] E\left[e^{R X_{j}}\right]=1
$$

Finally, we consider the aggregate loss up to time $t, L(t)=\sum_{j=1}^{N(t)} X_{j}-c t$ and the random variable $L=\max \{L(t), t \geq 0\}$ as the maximal aggregate loss, so that $\delta(u)=\operatorname{Pr}\{L \leq u) . L$ has a compound geometric distribution [see Dickson and Hipp (1998)].

We limit our work to the cases when $k(\cdot)$ is an exponential distribution or an Erlang $(2, \beta)$ distribution. The first case corresponds to the well known classical model, whereas the second case was treated recently by Dickson (1998) and Dickson and Hipp (1998). Our purpose in this paper is to find numerical solutions for the ruin/survival probabilities using Fourier/Laplace transforms and their properties, particularly with the use of an inversion formula for these transforms. We will consider the accuracy of the approximations by studying examples, although we don't have exact figures for one of them. In this latter case we will compare and discuss with another approximating method.

In the next section we present the Fourier/Laplace transform and its properties as well as the inversion formula for the transform, which we will use to obtain the numerical values for the ruin probabilities. In Section 3 we deal with the classical model and present some examples and compare the accuracy of the numerical figures obtained. In Section 4 we study the $\operatorname{Erlang}(2,2)$ renewal process, together with some examples. Section 5 presents the computation of the probability and severity of ruin in the classical model. The last section contains some concluding remarks about the method presented.

\section{THE TRANSFORM AND ITS PROPERTIES}

Let $f(x)$ be a continuous function defined for $x \geq 0$ whose integral exists for all $x>0$. Its Fourier transform is

$$
\bar{f}(i s)=\int_{0}^{\infty} e^{i s x} f(x) d x
$$

where $i=\sqrt{-1}$. We note that if $f(\bullet)$ is a density function then $\bar{f}(\bullet)$ is the corresponding characteristic function.

In this work we will need some properties dealing with these transforms. We write them down in what follows. These properties are easy to show, and we refer to Poularikas (1996), for instance.

Property 1 Let $f(\bullet)$ and $g(\bullet)$ be defined on $\mathcal{R}_{0}^{+}$as above and $h(x)=a f(x)+b g(x)$, where $a$ and $b$ are two constants. Then

$$
\bar{h}(i s)=a \bar{f}(i s)+b \bar{g}(i s)
$$


Property 2 Let $F(\cdot)$ be defined on $\mathcal{R}_{0}^{+}$as above, and let $\lim _{x \rightarrow \infty} F(x)=1, G(x)=$ $1-F(x), f(x)=F^{\prime}(x)$ and $g(x)=G^{\prime}(x)$. Then

$$
\bar{G}(i s)=\frac{i}{s}[1-F(0)-\bar{f}(i s)]=\frac{i}{s}[G(0)+\bar{g}(i s)] .
$$

Property 3 Let $\left\{f_{j}(\cdot)\right\}_{j=1}^{n}$ be functions defined on $\mathcal{R}_{0}^{+}$as above, and let $h(\bullet)$ be the $n$-th convoluted function, $h(x)=f_{1}{ }^{*} f_{2}{ }^{*} \ldots * f_{n}(x)$, where $f_{j}{ }^{*} f_{k}(x)=\int_{0}^{x} f_{j}(x-y)$ $f_{k}(y) d y$. Then

$$
h(i s)=\prod_{j=1}^{n} \bar{f}_{j}(i s) .
$$

The Fourier transform of $f(\bullet)$ can be split up into two parts considering the real and complex parts:

$$
\bar{f}(i s)=\operatorname{Re}(\bar{f}(i s))+i \operatorname{Im}(\bar{f}(i s))=\int_{0}^{\infty} \cos (s x) f(x) d x+i \int_{0}^{\infty} \sin (s x) f(x) d x .
$$

Consider now in the following the (Fourier) cosine transform and the inverse transform:

$$
\varphi(s)=\int_{0}^{\infty} \cos (s x) f(x) d x
$$

and

$$
\int_{0}^{x} f(y) d y=\lim _{z \rightarrow \infty} \frac{2}{\pi} \int_{0}^{z} \frac{\sin (x s)}{s} \varphi(s) d s,
$$

where $f(x)$ is a continuous non-negative function defined on $\mathcal{R}^{+}$, whose integral exists for all $x>0$. From the above we can write the following:

Result 1 Let $f(x)$ be a continuous non-negative function defined on $R_{0}^{+}$, whose integral exists for all $x>0$, and $f(x)=F^{\prime}(x)$. Then

$$
F(x)=F(0)+\lim _{z \rightarrow \infty} \frac{2}{\pi}\left[\int_{0}^{z} \frac{\sin (x s)}{s} \operatorname{Re}(\bar{f}(i s)) d s\right] .
$$

This result is the key to our future developments. The cosine transform and its inverse are well known. [See for instance Poularikas (1996); Garcia (2000) contains a more general proof]. We have examples of the use of this kind of inverse formula in the actuarial literature to compute ruin probabilities in the classical model. For this purpose Seal (1977) considered the expression

$$
f(x)=\frac{2 e^{c x}}{\pi} \int_{0}^{\infty} \cos (x s) \operatorname{Re}(\bar{f}(c+i s)) d s,
$$

where $c$ is an appropriate constant, in particular he put $f(x)=\psi(x)$ for the classical model. He then considered the trapezoidal quadrature of the integral 
above and particular positive values for the constant $c$ to compute numerical values for the ruin probability in the classical model [see Seal (1977)]. If we look at formula (1) we see that its derivative corresponds to (2) with $c=0$. Seal (1977) discarded the case $c=0$ in (2) saying that this case may be not applicable in his formula. Seal's (1977) expression for $\psi(x)$ has been recently retrieved by Usábel (2001) who used the mid-point integration rule with step $h=\pi / 2 u$ and parameter $c=A / 2 u$ to give

$$
\begin{aligned}
\psi(u) & =\frac{2 e^{c u}}{\pi} \int_{0}^{\infty} \cos (u s) \operatorname{Re}(\bar{\psi}(c+i s)) d s \\
& =\frac{e^{A / 2}}{2 u} \operatorname{Re}\left(\bar{\psi}\left(\frac{A}{2 u}\right)\right)+\frac{e^{A / 2}}{2 u} \sum_{j=1}^{\infty}(-1)^{j} \operatorname{Re}\left(\bar{\psi}\left(\frac{A}{2 u}+\left(\frac{j \pi}{u}\right) i\right)\right),
\end{aligned}
$$

and considered particular values for $A=15 \log 10 ; 20 \log 10$.

This latter author underlined the fact that it is not an easy task solving the integral in (3) numerically because the integrand is a rapidly oscillating function. This is, of course, also the case for computing the integral in (1). However, the use of our calculation method in our examples revealed good results. To compute numerically the integral in (1) we used the so called dicotomic approach algorithm explained in Garcia (1999), which is presented in comparison to Simpson's Rule and is reproduced in the Appendix. In the numerical computation we have used Visual Basic programming, as well the Mathematica package for some cases. In our applications we have considered a maximum error of $10^{-10}$ in the computation of the integral for each subdivision (see the Appendix). As a truncation procedure in the calculation of the integral for the next interval we have used the same value $10^{-10}$ as the maximum value to consider. As we will show we get good approximations with our method even for reasonably high values of the initial surplus.

We are mainly interested in calculating the probability of ultimate ruin in both the classical and in a non-classic model, although we can extend this method to other ruin problems. In the last section we consider the calculation of the probability and severity of ruin in the classical model as an example of this.

\section{RUIN PROBABILITY IN THE CLASSICAL MODEL}

In this section we consider the classical compound Poisson model, i.e. $K(t)=$ $1-\exp \{-\lambda t\}, t>0$. From Gerber (1979) we know that

$$
\psi^{\prime}(u)=\frac{\lambda}{c} \psi(u)-\frac{\lambda}{c} \int_{0}^{u} p(x) \psi(u-x) d x-\frac{\lambda}{c}[1-P(u)], u \geq 0 .
$$

From here we can calculate $\overline{\psi^{\prime}}(i s)$ as follows, using Properties 1 and 3

$$
\overline{\psi^{\prime}}(i s)=\frac{\lambda}{c} \bar{\psi}(i s)-\frac{\lambda}{c} \bar{p}(i s) \bar{\psi}(i s)-\frac{\lambda i}{c s}+\frac{\lambda i}{c s} \bar{p}(i s) .
$$


Now using Property 2 and rearranging we get

$$
\overline{\psi^{\prime}}(i s)=-\frac{\frac{\lambda i}{c s} \delta(0)[1-\bar{p}(i s)]}{1-\frac{\lambda i}{c s}[1-\bar{p}(i s)]}=-\frac{\frac{\lambda}{c s} \delta(0) \operatorname{Im}(\bar{p}(i s))+\frac{\lambda}{c s} \delta(0)[1-\operatorname{Re}(\bar{p}(i s))] i}{1-\frac{\lambda}{c s} \operatorname{Im}(\bar{p}(i s))-\frac{\lambda}{c s}[1-\operatorname{Re}(\bar{p}(i s))] i}
$$

We then can write

$$
\overline{\psi^{\prime}}(i s)=\frac{A+i B}{C-i D}=\frac{A C-B D+i(C B+A D)}{C^{2}+D^{2}}
$$

where

$$
\begin{aligned}
& A=-\frac{\lambda \delta(0)}{c s} \operatorname{Im}(\bar{p}(i s)), B=-\frac{\lambda}{c s} \delta(0)[1-\operatorname{Re}(\bar{p}(i s))], C=1-\frac{\lambda}{c s} \operatorname{Im}(\bar{p}(i s)), \\
& D=\frac{\lambda}{c s}[1-\operatorname{Re}(\bar{p}(i s))] .
\end{aligned}
$$

Expression (1) then becomes

$$
\psi(u)=\psi(0)+\lim _{z \rightarrow \infty} \frac{2}{\pi}\left[\int_{0}^{z} \frac{\sin (u s)}{s} \frac{A C-B D}{C^{2}+D^{2}} d s\right] .
$$

\section{Example 1 Exponential-Exponential model}

We consider $P(x)=1-\exp \{-\beta x\}$. For this case it is well known that $\psi(u)=\frac{\lambda}{\beta c}$ $\exp \{-(\beta-\lambda / c) u\}$. We get easily that $\operatorname{Re}(\bar{p}(i s))=\beta^{2} /\left(\beta^{2}+s^{2}\right)$ and $\operatorname{Im}(\bar{p}(i s)) \stackrel{\beta c}{=}$ $\beta s /\left(\beta^{2}+s^{2}\right)$. We set $\lambda=\beta=1$ and $c=1.1$ in the calculations.

Table 1 shows values for $\psi(u)$. The key for the table is the following: column (1) gives the exact figures, column (2) the approximating values from the application of formula (5), and the last column gives the ratio (1)/(2).

TABLE 1

$\psi(u)$ FOR EXPONENTIAL(1)-EXPONENTIAL(1) MODEL

\begin{tabular}{rccc}
\hline $\boldsymbol{u}$ & $(\mathbf{1})$ & $(\mathbf{2})$ & $(\mathbf{1}) /(\mathbf{2})$ \\
\hline 0 & 0.90909 & 0.90909 & 1.00000 \\
1 & 0.83009 & 0.83009 & 1.00000 \\
2 & 0.75796 & 0.75796 & 1.00000 \\
3 & 0.69209 & 0.69209 & 1.00000 \\
4 & 0.63195 & 0.63195 & 0.99999 \\
5 & 0.57703 & 0.57703 & 1.00000 \\
10 & 0.36626 & 0.36626 & 1.00000 \\
20 & 0.14756 & 0.14756 & 1.00000 \\
30 & 0.05945 & 0.05945 & 1.00001 \\
40 & 0.02395 & 0.02395 & 0.99991 \\
50 & 0.00965 & 0.00965 & 1.00021 \\
\hline \hline
\end{tabular}




\section{Example 2 Exponential-Gamma model}

We consider $p(x)=\frac{\beta^{\alpha}}{\Gamma(\alpha)} x^{\alpha-1} e^{-\beta x}$. We have

$$
\begin{aligned}
& \bar{p}(i s)=\left(\frac{\beta-i s}{\beta}\right)^{-\alpha}=\eta^{\alpha} e^{-i \alpha \vartheta}=\eta^{\alpha} \cos (\alpha \vartheta)-i \eta^{\alpha} \sin (\alpha \vartheta) \\
& \eta=\left(1+s^{2} / \beta^{2}\right)^{-1 / 2} \\
& \vartheta=\arccos (\eta),
\end{aligned}
$$

i.e. $\operatorname{Re}(\bar{p}(i s))=\eta^{\alpha} \cos (\alpha \vartheta)$ and $\operatorname{Im}(\bar{p}(i s))=\eta^{\alpha} \sin (\alpha \vartheta)$. From Egidio dos Reis (1993) we know that with $\alpha=2$

$$
\begin{aligned}
& \psi(u)=\sum_{k=1}^{2} \frac{\beta+r_{k}}{\beta\left(3 \beta+r_{k}\right)}\left(3 \beta+2 r_{k}\right) e^{r_{k} u} \text { and } \\
& r_{k}=\frac{1}{2}\left[\frac{\lambda}{c}-2 \beta+(-1)^{k} \sqrt{\left(\frac{\lambda}{c}\right)^{2}+4 \beta\left(\frac{\lambda}{c}\right)}\right], \quad k=1,2 .
\end{aligned}
$$

We set $\lambda=1, \beta=2$ and $c=1.1$. Table 2 shows values of $\psi(u)$ for this example. The key for the table is the same as in the previous case.

TABLE 2

$\psi(u)$ FOR EXPONENTIAL(1)-GAMma(2,2) MODEL

\begin{tabular}{rccc}
\hline \hline $\boldsymbol{u}$ & $\mathbf{( 1 )}$ & $\mathbf{( 2 )}$ & $\mathbf{( 1 ) / ( 2 )}$ \\
\hline 0 & 0.90909 & 0.90909 & 1.00000 \\
1 & 0.81269 & 0.81269 & 1.00000 \\
2 & 0.71942 & 0.71942 & 1.00000 \\
3 & 0.63649 & 0.63649 & 1.00000 \\
4 & 0.56311 & 0.56311 & 1.00000 \\
5 & 0.49819 & 0.49819 & 1.00000 \\
10 & 0.27001 & 0.27001 & 1.00000 \\
20 & 0.07932 & 0.07932 & 0.99998 \\
30 & 0.02330 & 0.02330 & 0.99992 \\
40 & 0.00684 & 0.00685 & 0.99962 \\
50 & 0.00201 & 0.00201 & 0.99833 \\
\hline \hline
\end{tabular}

\section{Example 3 Exponential-Pareto model}

We consider $P(x)=1-\left(\alpha_{2} /\left(\alpha_{2}+x\right)\right)^{\alpha_{1}}$. We set $\alpha_{1}=2 ; \alpha_{2}=1$ and again $\lambda=1$, $c=1.1$. In this case we don't have a closed form for the characteristic function of the Pareto density. This means that for the calculation of (5) we have to compute a double integral. This results in an increase in computer time. 
Table 3 shows values of $\psi(u)$ for this example. The key for the table is the following: (1) gives the approximations by Dickson et al (1995) and by Ramsay and Usábel (1997) $(u \geq 0)$, (2) shows our approximations and the last column the ratio (1)/(2). The values in column (1) for $u=30,50,500,1000$ were not available in Dickson et al (1995) so, we took them from Ramsay and Usábel (1997). Where available, figures from both authors give the same values to five decimal places. The approximations by Dickson et al (1995) are based on a discrete time compound Poisson model and the figures by Ramsay and Usábel (1997) have been produced via product integration. In this example we extended our calculation to values of $u$ greater than 50 , so that we could compare smaller values of $\psi(u)$, say, closer to $1 \%$. So, we can deduce the quality of approximations in all examples when we have smaller ruin probabilities.

TABLE 3

$\psi(u)$ For EXPONENTIAL(1)-PARETO(2,1) MODEL

\begin{tabular}{rccc}
\hline $\boldsymbol{u}$ & $\mathbf{( 1 )}$ & $\mathbf{( 2 )}$ & $\mathbf{( 1 ) / ( 2 )}$ \\
\hline 0 & 0.90909 & 0.90909 & 1.00000 \\
2 & 0.81023 & 0.81023 & 1.00000 \\
4 & 0.74976 & 0.74976 & 1.00000 \\
10 & 0.62713 & 0.62713 & 1.00000 \\
20 & 0.49814 & 0.49814 & 1.00000 \\
30 & 0.41144 & 0.41144 & 1.00000 \\
40 & 0.34789 & 0.34790 & 0.99999 \\
50 & 0.29916 & 0.29916 & 0.99999 \\
100 & 0.16486 & 0.16486 & 1.00000 \\
500 & 0.02512 & 0.02512 & 1.00012 \\
1000 & 0.01134 & 0.01135 & 0.99921 \\
\hline \hline
\end{tabular}

RUIN PROBABILITY IN THE ERLANG $(2, \beta)$ MODEL

In this section we consider the model in Dickson (1998) and Dickson and Hipp (1998), i.e. $k(t)=\beta^{2} t \exp \{-\beta t\}, t>0$. From Dickson and Hipp (1998) we have that

$$
c^{2} \delta^{\prime \prime}(u)-2 \beta c \delta^{\prime}(u)+\beta^{2} \delta(u)=\beta^{2} \int_{0}^{u} p(x) \delta(u-x) d x,
$$

or equivalently

$$
c^{2} \psi^{\prime \prime}(u)-2 \beta c \psi^{\prime}(u)+\beta^{2} \psi(u)=+\beta^{2}[1-P(u)]+\beta^{2} \int_{0}^{u} p(x) \psi(u-x) d x .
$$

If we follow the method by Dickson and Hipp (1998, Section 2) for Laplace transforms, applying the properties in Section 2 we get that

$$
\overline{\psi^{\prime}}(i s)=\frac{\beta^{2} \delta(0)[1-\bar{p}(i s)]-c^{2} s \psi^{\prime}(0) i}{2 \beta c s i-c^{2} s^{2}+\beta^{2}[1-\bar{p}(i s)]} .
$$


On the other hand, we have that for the aggregate loss distribution function $\delta(u), E\left[e^{i s L}\right]=\delta(0)-\overline{\psi^{\prime}}(i s)$ and knowing that $\lim _{s \rightarrow 0} E\left[e^{i s L}\right]=1$ we get that $c^{2} \psi^{\prime}(0) i$ $=2 \beta c i-\beta^{2} p_{1} i-2 \beta c \delta(0) i$. Then

$$
\begin{aligned}
\overline{\psi^{\prime}}(i s) & =\delta(0)-\frac{2 \beta c s i-c^{2} s^{2} \delta(0)-s \beta^{2} p_{1} i}{2 \beta c s i-c^{2} s^{2}+\beta^{2}[1-\operatorname{Re}(\bar{p}(i s))]-\beta^{2} \operatorname{Im}(\bar{p}(i s)) i} \\
& =\left(\delta(0)+\frac{A C+B D}{C^{2}+D^{2}}\right)+\frac{B C-A D}{C^{2}+D^{2}} i,
\end{aligned}
$$

where $A=c^{2} s^{2} \delta(0), B=s \beta^{2} p_{1}-2 \beta c s, C=\beta^{2}[1-\operatorname{Re}(\bar{p}(i s))]-c^{2} s^{2}$ and $D=2 \beta c s-$ $\beta^{2} \operatorname{Im}(\bar{p}(i s))$. Hence, from (1) we have

$$
\begin{aligned}
& \psi(u)=\psi(0)+\lim _{z \rightarrow \infty} \frac{2}{\pi}\left[\int_{0}^{z} \frac{\sin (u s)}{s}\left(\delta(0)+\frac{A C+B D}{C^{2}+D^{2}}\right) d s\right] \\
& =\psi(0)+\delta(0) \operatorname{sgn}(u)+\lim _{z \rightarrow \infty} \frac{2}{\pi}\left[\int_{0}^{z} \frac{\sin (u s)}{s} \frac{A C+B D}{C^{2}+D^{2}} d s\right],
\end{aligned}
$$

where $\operatorname{sgn}(\cdot)$ is the sign or signum function.

In expression (7) we need to compute $\delta(0)$. Dickson and Hipp (1998) show that for this process

$$
\delta(0)=\frac{2 \beta c-\beta^{2} p_{1}}{c^{2} s_{0}}
$$

where $s_{0}$ is the unique positive root of the equation in real $s, c^{2} s^{2}-2 \beta c s+\beta^{2}(1-$ $\vec{p}(-s))=0$.

\section{Example 4 Erlang(2,2)-Exponential model}

Let $p(\bullet)$ be an Exponential distribution with mean $\theta^{-1}$. For this case it can be shown easily that $\psi(u)=\psi(0) \exp \{-R u\}, \psi(0)=(\theta-R) / \theta$ and $R=(\theta c-2 \beta+$ $\left.\sqrt{\theta^{2} c^{2}+4 \theta \beta c}\right) / 2 c$ is the adjustment coefficient. We set $\beta=2, c=1.1$ and $\theta=1$. Table 4 shows values of $\psi(u)$ for this example. The key for the table is the same as in Table 1.

\section{Example 5 Erlang(2,2)-Erlang(2,2) model}

We set $\beta=2, c=1.1$ and let $p(\bullet)$ be an $\operatorname{Erlang}(2,2)$ distribution. For this case we have that $\psi(u)=0.88407524 e^{-0.18181818 u}-0.01085889 e^{-2.78924038 u}$ [see Dickson (1998)]. Table 5 shows values of $\psi(u)$ for this example. The key for the table is the same as above.

\section{Example 6 Erlang(2,2)-Pareto(2,1) model}

We consider a Pareto(2,1) claim amount. Again $\beta=2, c=1$.1. In this case we don't have exact results for the ruin probability $\psi(u)$, however we can compare 
TABLE 4

$\psi(u)$ FOR ERLANG(2,2)-EXPONENTIAL(1) MODEL

\begin{tabular}{rccc}
\hline \hline $\boldsymbol{u}$ & $\mathbf{( 1 )}$ & $\mathbf{( 2 )}$ & $\mathbf{( 1 ) / ( 2 )}$ \\
\hline 0 & 0.88006 & 0.88006 & 1.00000 \\
5 & 0.48315 & 0.48315 & 1.00000 \\
10 & 0.26524 & 0.26524 & 1.00000 \\
15 & 0.14561 & 0.14561 & 1.00000 \\
20 & 0.07994 & 0.07994 & 0.99998 \\
25 & 0.04389 & 0.04389 & 1.00002 \\
30 & 0.02409 & 0.02409 & 0.99993 \\
\hline \hline
\end{tabular}

TABLE 5

$\psi(u)$ FOR ERLANG(2,2)-ERLANG(2,2) MODEL

\begin{tabular}{rccc}
\hline \hline $\boldsymbol{u}$ & (1) & (2) & (1)/(2) \\
\hline 0 & 0.87322 & 0.87322 & 1.00000 \\
5 & 0.35619 & 0.35619 & 1.00000 \\
10 & 0.14350 & 0.14350 & 1.00000 \\
15 & 0.05782 & 0.05782 & 1.00000 \\
20 & 0.02329 & 0.02329 & 0.99995 \\
25 & 0.00938 & 0.00938 & 1.00000 \\
30 & 0.00378 & 0.00378 & 0.99950 \\
\hline \hline
\end{tabular}

approximations obtained from different methods. We can take Dickson \& Hipp's (1998, Section 5) suggestion and consider upper and lower bounds using "Method 1" of Dufresne \& Gerber (1989) and average these like Dickson et al. (1995) do. Like in Example 3 we show figures for $\psi(u)$ for higher values of the initial surplus.

Table 6 shows values of $\psi(u)$ for this example. The key for the table is the following: (1) and (2) show the lower and upper bound, respectively, (3) shows the average between (1) and (2), (4) shows our approximating values. We note that for our approximation for $u=500,1000$ does not look so accurate. Actually, they come slightly outside the interval delimited by the values in columns (1) and (2). However, we must note that the bounds in this example require some numerical integral calculation, which may have a greater negative effect on the computations in columns (1) and (2) for very high $u$ 's. See Dickson and Hipp (1998, Example 7).

\section{THE PROBABILITY AND SEVERITY OF RUIN}

The method for computing ultimate ruin probabilities presented in previous sections can also be applied to other problems in ruin theory. Furthermore, it 
TABLE 6

$\psi(u)$ For ErLANG(2,2)-PAReto(2,1) MODEL

\begin{tabular}{rcccc}
\hline $\boldsymbol{u}$ & (1) & (2) & (3) & (4) \\
\hline 0 & 0.88664 & 0.88664 & 0.88664 & 0.88664 \\
5 & 0.69884 & 0.69941 & 0.69912 & 0.69912 \\
10 & 0.60109 & 0.60171 & 0.60140 & 0.60140 \\
15 & 0.53007 & 0.53070 & 0.53039 & 0.53038 \\
20 & 0.47425 & 0.47485 & 0.47455 & 0.47455 \\
25 & 0.42858 & 0.42915 & 0.42886 & 0.42886 \\
30 & 0.39028 & 0.39082 & 0.39055 & 0.39055 \\
40 & 0.32935 & 0.32983 & 0.32959 & 0.32961 \\
50 & 0.28294 & 0.28336 & 0.28315 & 0.28315 \\
100 & 0.15635 & 0.15657 & 0.15646 & 0.15645 \\
500 & 0.02473 & 0.02474 & 0.02474 & 0.02472 \\
1000 & 0.01127 & 0.01128 & 0.01128 & 0.01124 \\
\hline \hline
\end{tabular}

can also be used in other areas of applied probability, e.g. queueing theory. In this section we consider its application to the probability and severity of ruin. We define the probability and severity of ruin (defective) distribution function, $G(u, x)$, as

$$
G(u, x)=\operatorname{Pr}\{T<\infty \text { and } U(T)>-x \mid U(0)=u\}, \quad x>0
$$

and let $g(u, x)$ be the associated density function.

We consider in this section the classical model only, i.e $k(t)=\lambda \exp \{-\lambda t\}$, $t>0$, however, extensions can be made. From Gerber et al (1987) we find the transform

$$
\bar{g}(i s, x)=\frac{\frac{\lambda}{c} e^{-i s x} \int_{x}^{\infty} e^{i s u}[1-P(u)] d u}{1-\frac{\lambda}{c} \int_{0}^{\infty} e^{i s u}[1-P(u)] d u}
$$

We write here $\bar{g}(i s ; x)$ to emphasize that the transform is obtained over the argument $u$. We can rewrite the formula as

$$
\begin{aligned}
\bar{g}(i s, x) & =\frac{\cos (s x) C(x, s)+\sin (s x) S(x, s)+i[\cos (s x) S(x, s)-\sin (s x) C(x, s)]}{c / \lambda-C(0, s)-i S(0, s)} \\
& =\frac{A C-B D}{C^{2}+D^{2}}+i \frac{B C+A D}{C^{2}+D^{2}}
\end{aligned}
$$

where $C(x, s)=\int_{x}^{\infty} \cos (s u)[1-P(u)] d u, S(x, s)=\int_{x}^{\infty} \sin (s u)[1-P(u)] d u, \mathrm{~A}=\cos$ $(s x) C(x, s)+\sin (s x) S(x, s), B=\cos (s x) S(x, s)-\sin (s x) C(x, s), C=c / \lambda-C(0, s)$ and $D=S(0, s)$. 
Knowing that $g(\infty, x)=0$ we get applying Property 2 that

$$
\overline{\frac{d}{d u}} g(i s, x)=-i s \bar{g}(i s ; x)-g(0, x)
$$

so that

$$
\begin{aligned}
& g(u, x)=g(0, x)+\lim _{z \rightarrow \infty} \frac{2}{\pi} \int_{0}^{z} \sin (u s)\left[\frac{B C+A D}{C^{2}+D^{2}}-\frac{g(0, x)}{s}\right] d s \\
& =g(0, x)(1-\operatorname{sgn}(u))+\lim _{z \rightarrow \infty} \frac{2}{\pi} \int_{0}^{z} \sin (u s) \frac{B C+A D}{C^{2}+D^{2}} d s .
\end{aligned}
$$

From here we get $G(u, x)=\int_{0}^{x} g(u, t) d t$.

In what follows we consider an example and compute figures for $G(u, x)$, when we have $\operatorname{Gamma}(2,2)$ claim amounts.

Example 7 Gamma(2,2) claim amounts

For a general $\operatorname{Gamma}(2, \beta)$ we get for $C(x, s)$ and $S(x, s)$ :

$$
\begin{aligned}
& C(x, s)=\frac{e^{-\beta x}\left[\beta^{2}\left(2 \beta+\beta^{2} x+s^{2} x\right) \cos (s x)-s\left(3 \beta^{2}+s^{2}+\beta^{3} x+\beta s^{2} x\right) \sin (s x)\right]}{\left(\beta^{2}+s^{2}\right)^{2}} \\
& S(x, s)=\frac{e^{-\beta x}\left[\beta^{2}\left(2 \beta+\beta^{2} x+s^{2} x\right) \sin (s x)+s\left(3 \beta^{2}+s^{2}+\beta^{3} x+\beta s^{2} x\right) \cos (s x)\right]}{\left(\beta^{2}+s^{2}\right)^{2}}
\end{aligned}
$$

Putting $\beta=2$ we get using the methods of Gerber et al (1987) that $g(u, x)=$ $\frac{\lambda}{c} e^{-2 x}\left(\vartheta_{1} e^{r_{1} u}+\vartheta_{2} e^{r_{2} u}\right)$, where $r_{1}=-0.1225022, r_{2}=-2.9684067$ and $\vartheta_{j}=(-1)^{j}(4 x$ $\left.+4+(2 x+1) r_{j}\right) /\left(r_{2}-r_{1}\right), j=1,2$.

Table 7 show figures for $G(u, x)$ with $x=1,2$. The key for this table is as in the previous examples.

\section{CONCLUDING REMARKS}

The technique introduced in this paper provides an easy way to obtain figures for ruin problems not only in the traditional model but also for other models, shown here in the case of an Erlang(2) risk model. In all the examples where exact figures are available, we get good approximation figures for the problems presented, up to reasonably high values of the initial surplus. Where exact values are not available we compare figures with the ones from existing methods which are believed to be producing good figures. With very high values of $u$ the numerical integration becomes unstable in contrast with the method by Usábel (2001). Here, we were mostly concerned in showing a simple method that could obtain relevant figures in many cases. Besides, we extended this method to other models. 
TABLE 7

$G(U, X)$ FOR EXPONENTIAL(1)-GAMMA(2,2)MODEL

\begin{tabular}{rcccccc}
\hline \hline & \multicolumn{3}{c}{$\boldsymbol{x = 1}$} & & \multicolumn{3}{c}{$\boldsymbol{x = 2}$} \\
$\boldsymbol{u}$ & $\mathbf{( 1 )}$ & $\mathbf{( 2 )}$ & $\mathbf{( 1 ) / ( 2 )}$ & $\mathbf{( 1 )}$ & $\mathbf{( 2 )}$ & $\mathbf{( 1 ) / ( 2 )}$ \\
\hline 0 & 0.66303 & 0.66303 & 1.00000 & 0.85914 & 0.85914 & 1.00000 \\
1 & 0.62874 & 0.62874 & 1.00000 & 0.77778 & 0.77778 & 1.00000 \\
2 & 0.55842 & 0.55842 & 1.00000 & 0.68902 & 0.68902 & 1.00000 \\
3 & 0.49414 & 0.49414 & 1.00000 & 0.60962 & 0.60962 & 1.00000 \\
4 & 0.43718 & 0.43718 & 1.00000 & 0.53934 & 0.53934 & 1.00000 \\
5 & 0.38677 & 0.38677 & 1.00000 & 0.47715 & 0.47715 & 1.00000 \\
10 & 0.20963 & 0.20963 & 1.00000 & 0.25861 & 0.25861 & 1.00000 \\
20 & 0.06158 & 0.06158 & 1.00000 & 0.07597 & 0.07597 & 1.00000 \\
30 & 0.01809 & 0.01809 & 1.00000 & 0.02232 & 0.02232 & 1.00000 \\
40 & 0.00531 & 0.00531 & 0.99999 & 0.00656 & 0.00656 & 1.00000 \\
50 & 0.00156 & 0.00156 & 1.00000 & 0.00193 & 0.00193 & 0.99999 \\
\hline \hline
\end{tabular}

Most of the work in ruin theory has been centred on the classical compound Poisson model. In recent times concerns started to include other renewal models, which allow contagion between the claims. We show in Section 4 that our method can also compute figures for some kind of these models, namely the Erlang $(2, \beta)$. It depends on having an expression for $\overline{\psi^{\prime}}(i s)$ (for Section 5, $\frac{\frac{d}{d u}}{d u}(i s, x)$, for other problems a corresponding formula). Dickson (1998) shows that we can get these expressions for $\operatorname{Erlang}(n, \beta)$, where $n$ is a positive integer. We have shown two of these cases, namely for $n=1,2$. The procedures for our problems, ultimate ruin probabilities or severity of ruin, will be similar for other $n$.

Finally, an additional remark on our computational work. We have programmed all examples with Visual Basic. In a few cases we also produced figures with Mathematica, in most of them for checking numbers only. Our Visual Basic programs revealed to be quite fast and producing good figures.

\section{REFERENCES}

Dickson, D.C.M. (1998) On a class of renewal risk processes, North American Actuarial Journal, 2(3), 60-73.

DICKSON, D.C.M. and HIPP, C. (1998) Ruin probabilities for Erlang(2) risk processes, Insurance: Mathematics and Economics, 22, 251-262.

Dickson, D.C.M., EGídio dos ReIs, A.D. and Waters, H.R. (1995) Some stable algorithms in ruin theory and their applications, Astin Bulletin, 25(2), 153-175.

DUfRESNE, F. and GerBer, H.U. (1989) Three methods to calculate the probability of ruin, Astin Bulletin, 19, 71-90.

Egidio Dos ReIS, A.D. (1993) How long is the surplus below zero?, Insurance: Mathematics and Economics, 12, 23-38. 
GARCIA, J.M.A. (1999) Characteristic functions and their application to risk processes, Cemapre Working Paper no. 6/99, ISEG.

GARCIA, J.M.A. (2000) Other properties of the cosine transform, Cemapre Working Paper no. 6/99, ISEG.

Gerber, H.U. (1979) An Introduction to Mathematical Risk Theory, S.S. Huebner Foundation for Insurance Education, University of Pennsylvania, Philadelphia.

Gerber, H.U., GoOvaerTs, M.J. and KAAS, R. (1979) On the probability and severity of ruin, Astin Bulletin 17, 151-163.

Poularikas, A. (1996) The Transforms and Applications Handbook, CRC Press.

RAMSAY, C.M. and USABEL, M.A. (1997) Calculating ruin probabilities via product integration, Astin Bulletin, 27(2), 263-271.

SEAL, H. (1977) Numerical inversion of characteristic functions, Scandinavian Actuarial Journal, $48-53$.

Sparre Andersen, E. (1957) On the collective theory of risk in the case of contagion between the claims, Transactions of the XV International Congress of Actuaries, 2, 219-229.

UsÁBEL M.R. (2001) Ultimate ruin probabilities for generalized Gamma-convolutions claim sizes, Astin Bulletin, 31(1), 59-79.

FÁTIMA D.P. LIMA

SGF - Sociedade Gestora de Fundos de Pensões

Lisbon

E-mail:flima@sgf.pt.

JORGE M.A. GARCIA

SGF - Sociedade Gestora de Fundos de Pensões

Lisbon

E-mail: jorgegarcia@sgf.pt.

Alfredo D. Edidio dos ReIS

Department of Mathematics

Technical University of Lisbon

Rua do Quelhas 6

1200-781 Lisboa

Portugal

Tel.: $+351-213925867$

Fax: $+351-213922781$

E-mail: alfredo@iseg.utl.pt. 


\section{APPENDIX}

\section{Simpson's Rule}

Let $y=f(x)$ be a continuous function in a closed interval $[a, b]$. Once that function is integrable in this interval, we can perform the numerical calculation of the corresponding integral using Simpson's Rule (SR):

$$
\int_{a}^{b} f(x) d x \simeq \frac{b-a}{6}[f(a)+4 f(m)+f(b)]-\frac{(b-a)^{5}}{2880} f^{(i v)}(c),
$$

where $m=(a+b) / 2$ is the midpoint of the interval and $c$ is another point of $[a, b]$. The General Simpson's Rule (GSR) generalizes the above expression considering a subdivision of the integrand interval into $2 n$ equal parts, giving

$$
\int_{a}^{b} f(x) d x \cong \frac{b-a}{6 n}\left[f(a)+4 \sum_{k=1}^{n} f_{2 k-1}+2 \sum_{k=1}^{n-1} f_{2 k}+f(b)\right] .
$$

We have considered $f_{k}=f(a+k h)$ and $h=(b-a) / 2 n$. The absolute error committed using (A2) is not greater than $\left|\frac{(b-a)^{5}}{2880 n^{4}}\right| f^{(i v)}(d)$, where $d$ is a certain point of the interval $[a, b]$.

\section{Dicotomic Approach}

Consider a subinterval $\left[a_{k}, b_{k}\right] \subset[a, b]$ and its midpoint $m_{k}$. Using GSR evaluate the following three integrals:

$$
I_{k}=\int_{a_{k}}^{b_{k}} f(x) d x, \quad L_{k}=\int_{a_{k}}^{m_{k}} f(x) d x \text { and } R_{k}=\int_{m_{k}}^{b_{k}} f(x) d x .
$$

If $\left|I_{k}-L_{k}-R_{k}\right|>\varepsilon_{k}$, where $\varepsilon_{k}$ is the maximum error admitted for a subdivision of order $k$, we take a new subdivision of the left-hand half interval $\left[a_{k}, m_{k}\right]$, which will be the interval $\left[a_{k+1}, b_{k+1}\right]$ for a new iteration. This procedure must be repeated until the relation $\left|I_{n}-L_{n}-R_{n}\right|<\varepsilon_{n}$ holds for some order $n>k$. At that point no more subdivisions of this interval are needed and $I_{n}$ should be considered as a parcel of the original integral. Once determined the value of the left-hand side of any interval, it is necessary to evaluate the integral of the right-hand side of the same interval.

The algorithm is simple but we must record that portion of the original interval that we are considering per iteration. This can be done by a binary tree where each node (an interval) has two sub-nodes: the left son corresponding to the left-hand subinterval and the right son to the right-hand subinterval. The root of the tree is the original interval with which the algorithm must start and the leaves are the sub-intervals that we must take into account to get the final value of the integral. 